Editorial

\title{
Editorial: etiology, prevention and prediction of preterm birth- what is new?
}

Volume 8 Issue 3 - 2017

\section{Editorial}

Preterm birth (PTB) is a significant global health issue and a major public health problem worldwide. It's the leading cause of perinatal morbidity and mortality. Despite this, the incidence of PTB has not changed during the last 50 years. Every year about 15 million babies are born preterm. PTB usually affects 5-7 \% of births, but they are estimated to be substantially higher in developing countries. ${ }^{1}$ The incidence of PTB has increased worldwide. In 2010, $11 \%$ of all live births were born preterm. ${ }^{2}$ Decreasing the incidence of prematurity, remains a universal public health goal.

PTBs are the births before 37 completed weeks of gestation. It is a major determinant of neonatal mortality and morbidity and has longterm adverse consequences for health. ${ }^{3,4}$ PTB is a complex syndrome with multifactorial aetiologies that can be classified into two groups: spontaneous PTBs (70\% of all PTB) and iatrogenic PTBs (30\% of all PTB). ${ }^{5}$

To avoid serious neonatal complications and consequences, early identification the pregnant woman at risk for PTB, timely implementation of appropriate intervention and treatment are crucial. In developed countries PTBs are responsible for about half of all neonatal deaths. Children who are born prematurely have higher rates of cerebral palsy, sensory deficits, learning disabilities and respiratory illnesses compared with children born at term. ${ }^{6}$ The morbidity associated with PTB often extends to later life, resulting in enormous physical, psychological and economic costs. ${ }^{7}$

PTB rates have not decreased in the last 50 years and in most developed countries continue to rise despite advances in knowledge of the risk factors and mechanisms related to preterm labour. Factors possibly contributing to but not completely explaining this upward trend include increasing rates of multiple births, greater use of assisted reproduction techniques, increases incidence of births among women over 34 years of age, changes in clinical practices, etc. Changes in the definitions of fetal loss, stillbirth and early neonatal death may also have contributed to the substantial increases in PTB rates recorded in developed countries in the past two decades. ${ }^{8,9}$

From a pathophysiological perspective, PTB is a highly complex and incompletely understood syndrome. In most cases, the causes of PTB are not diagnosed, and the etiology is likely to be multifactorial. Risk factors for PTB can be classified as: maternal characteristics, reproductive history and characteristics of the actual pregnancy. PTB is multifactorial disorder which creation involves various exogenous and endogenous risk factors whose interactions initiated early, start asynchronous delivery mechanism. PTB is now thought to be a syndrome initiated by multiple mechanisms, including infection/ inflammation, uteroplacental ischaemia or haemorrhage, placental malperfusion, Mullerian anomalies, uterine overdistension, stress, and other immunologically mediated processes ${ }^{9-14}$ Pregnant outside marriage and without antenatal care are at higher risk for PTB. These conditions are often associated with low socioeconomic status, smoking, alcohol or drug abuse and poor nutritional status all

\author{
Emilija Jasovic-Siveska \\ Specialist of Gynecology and Obstetrics at PHO Medihelp \\ Bitola, Macedonia
}

Correspondence: Emilija Jasovic-Siveska, MD, PhD, Specialist of Gynecology and Obstetrics at PHO Medihelp Bitola, Professor of Gynecology and Obstetrics at University St Clement of Ohrid, Bitola, Macedonia, Tel 38972222256 , Email medihelp@t-home.mk

Received: December 25, 2017 | Published: December 28, 2017

independent risk factors of PTB as well. ${ }^{10}$ Other maternal risk factors are: psychological and social stress or depression, genetic factors, inflammatory and immune response, cervical insufficiency, etc. ${ }^{15,16}$ Baer et al. ${ }^{17}$ report that a change in partner seemed to be protective against PTB. They concluded that more research into the genetic and immune influences of change inpartner and preterm birth is needed.

About $50 \%$ of all preterm delivery occurs in primiparous women. Previous PTB is actually the strongest single risk factor. It has been reported that risk of PTB ranges from $15 \%$ to more than $50 \%$, depending on the number of previous preterm deliveries (for each previous preterm delivery the risk of a subsequent PTB increases). In some studies the induced abortions increase the risk of PTB due to cervical damage during termination of pregnancy. ${ }^{18}$

Twin births have become more prevalent in developed countries over the last decades. In 2014, the rate of twin birth in the USA was 33.9/1000 live births. Multiple pregencies are at increased risk of maternal, perinatal and infant morbidity and mortality. Multiple gestations have an increased risk for PTB, and result in $12-27 \%$ of all preterm deliveries. In the past decades there was a great increase in the incidence of multiple deliveries, above all as a result of the use of assisted reproduction technologies. ${ }^{19,20}$

At least $40 \%$ of preterm births are associated with intrauterine infection. 3 In individual cases it is often difficult to determine whether infection is the cause or consequence of the processes leading to preterm delivery. However, there is abundant evidence that the infection and the inflammation generated by the infection, whether within the gestational tissues or elsewhere, is a primary cause of a substantial proportion of preterm births. The incidence of genital tract infections are about $25-40 \%$ of PTBs. Women with Chlamydia trachomatis, Gardnerella Vaginalis, Trichomonas Vaginalis, Neisseria Gonorrhoeae, Treponema Pallidum, have a higher rate of preterm births. Still, the infection is difficult to detect due to the limitations of conventional microbial techniques and the difficulties in obtaining 
appropriate diagnostic samples during pregnancy. Spontaneous PTBs that occur before the 34 th week of gestation are strongly associated with intrauterine infection. ${ }^{9,21}$

Experimental models also suggest the possible induction of labor by viral infection. Polyinosinic: cytidylic acid [poly (I:C)] is a tolllike receptor (TLR) 3 ligand and a synthetic analog of double-stranded RNA (a replication intermediate in the life cycle of most viruses). Poly (I:C) induces preterm delivery in mice when injected into the uterus in mid-late gestation or systemically in late gestation. ${ }^{22}$

To further investigate the relationship between intrauterine infection and fetal inflammation, Yoon and colleagues examined putative relationships between umbilical cord blood IL-6 concentration, funisitis, amniotic-fluid microbial culture, and neonatal sepsis in a cohort of 315 preterm (20-35 weeks' gestation) infants. Preterm infants with funisitis were found to have higher cord blood IL-6 concentrations than those without funisitis. Fetal plasma IL-6 levels have also been shown to be significantly associated with inflammatory lesions in the chorioamnion. ${ }^{23,24}$

The history of vaginal bleeding at any time of pregnancy is associated with PTB and also other adverse perinatal outcomes. Vaginal bleeding is a manifestation of decidual damage, but can also be idiopathic. It may be a sign of retro placental haematoma detected by ultrasound examination in the first trimester. Placental abruption is a major obstetrical emergency. General risk factors for placental abruption are maternal smoking, use of alcohol, placenta preavia, preeclampsia and chorionamnionitis. ${ }^{9,25,26}$

Accurate prediction of PTB among asymptomatic pregnant women and those with threatened PTB might offer an opportunity to target more intensive antenatal surveillance and prophylactic measures to those most likely to benefit from primary, secondary or tertiary prevention. Primary prevention is prevention of the onset of spontaneous PTB in asymptomatic women by cessation of smoking and/or alcohol use, by maintaining a healthy genitourinary tract and periodontal status or with administration of progestational agents, or application of cervical cerclage. Secondary prevention involves steps that can be taken to attenuate, stop or reverse the progress of spontaneous PTB in its early stages, before advanced cervical dilatation by using tocolytic agents. Tertiary prevention is prevention of neonatal complications connected with prematurity by using antenatal corticosteroids for artificial fetal lung maturity. ${ }^{9}$

In the 1990s, two biologic markers were discovered that improve the precision of preterm birth risk assessment: fetal fibronectin and transvaginal ultrasonography. ${ }^{26,27}$

The best method of cervical evaluation is transvaginal ultrasonography, rather than digital palpation. The measurement of cervical length at 20 to 24 weeks is the first marker for identification of the cervical incompetence. In women who had a previous PTB or second-trimester loss, cervical cerclage is either carried out electively in the first trimester, or it is reserved for those where serial scans, starting from the first-trimester, demonstrate cervical shortening. At 11 to 13 weeks, the endocervical length in pregnancies complicated by subsequent spontaneous delivery before 34 weeks was shorter $(<25 \mathrm{~mm})$ than in those delivering after 34 weeks and the risk of early delivery was inversely related to cervical length. ${ }^{26-28}$

Biomarkers in serum or plasma are easy to collect, with minimal discomfort to the patient. Alpha fetoprotein, ferritin, C-reactive protein, various cytokines and relaxin are examples of biomarkers that can be measured in serum \& plasma, and are used for prediction of PTB. Inflammation-associated proteins are produced in response to inflammation in the choriodecidual space and also in extra-uterine tissues. Proteins of the choriodecidua are thought to leak into the amniotic fluid, plasma or into cervical and vaginal fluid from the placenta or choriodecidual space as a result of tissue disruption. Some proteins, IGFBP-1, FFN and prolactin, may have no actual role in the pathway leading to PTB, but may serve as predictors. However, a systematic review reported by Polettini et al sown no single or combination of biomarkers could be identified to best predict PTB risk. This systematic review included 10 studies. There was included 42 PTB- related proteins, with RANTES (Regulated on Activation, Normal T Cell Expressed and Secreted) and IL-10 folowed by MIP$1 \beta$, GM-CSF, eotaxin and TNF receptor $1 .^{29}$

\section{Preventing Tools for PTB}

Cervical cerclage: It has been one of the preventive strategies used for many years, but there are no studies that show overall evidence except in very specific cases. The literature shows evidence that cerclage provides clear and proven benefits only in circumstances diagnosed with cervical incompetence. Cerclage may have a beneficial effect in preventing preterm delivery when there is a history of PTB and an objective decrease in cervical length or increase cervix dilatation in non-symptomatic patients. In cases with uterine abnormalities and multiple pregnancies, cerclage has failed to show evidence of improvement in perinatal results. In singleton gestations without prior spontaneous PTB but with transvaginal cervicometry less than $25 \mathrm{~mm}$ in the second trimester, cerclage does not seem to prevent PTB or improve neonatal outcome. ${ }^{30}$

Progesterone: The optimal progesterone formulation, route of delivery, and dose for the prevention of PTB has not yet been determined. The majority of such clinical trials were performed with diverse formulations of progesterone (vaginal route or intramuscular injection of 17 OHP-C). The most part of randomized subjects is represented by women with a history of at least one previous spontaneous PTB or by multiple pregnancies. Asymptomatic midsecond trimester women with a very short cervix as well as thirdtrimester patients having had a successful treatment of a preterm labor episode were also admitted to 'progesterone' supplementation. Micronized progesterone capsules ( $200 \mathrm{mg}$ vaginally daily) were used in asymptomatic women with a very short cervix (less than $15 \mathrm{~mm}$ ), and appeared to be effective for such an indication. Supplemental 17 OHPC treatments does not benefit women with short cervix and previous preterm birth submitted to cervical cerclage for suspected cervical insufficiency. ${ }^{31-33}$

Clinical predictors of PTB in 2017 are similar to those mentioned in the last decades. The delivery of a preterm infant brings considerable health care costs which are strongly gestational age-dependent. The costs are not just those incurred while in the hospital's neonatal intensive care unit. Some health problems that develop at this time can persist for years, leading to long-lasting use of healthcare and social services, including special education and rehabilitation for those with physical handicaps. Maternal hospitalization before and after delivery and an increased number of Caesarean sections also increase costs. Reduction of the risk of PTB among asymptomatic pregnant women and those symptomatic with threatened preterm labour may offer the opportunity to target care at those most likely to benefit. $5,6,9$

All therapies and strategies should improve outcomes for the baby, not only prevent PTB. Little research has assessed perinatal mortality or longer term outcomes since this is difficult and expensive. Funding bodies must resource the collection of these outcomes. Until then, preterm births will remain a major public health issue world-wide. ${ }^{9,31,34}$ 


\section{Acknowledgements}

None.

\section{Conflicts of Interest}

None.

\section{References}

1. Lawn JE, Cousens SN, Darmstadt GL, et al. One year after The Lancet Neonatal Survival Series-was the call for action heard? Lancet. 2006;367(9521):1541-1547.

2. Blencowe H, Cousens S, Oestergaard MZ, et al. National, regional, and worldwide estimates of preterm birth rates in the year 2010 with time trends since 1990 for selected countries: a systematic analysis and implications. Lancet. 2012;379(9832):2162-2172.

3. Goldenberg RL, Culhane JF, Iams JD, et al. Epidemiology and causes of preterm birth. Lancet. 2008;371(9606):75-84.

4. Wang ML, Dorer DJ, Fleming MP, et al. Clinical outcomes of near-term infants. Pediatrics. 2004;114(2):372-376.

5. Saigal S, Doyle LW. An overview mortality and sequelae of preterm birth from infancy to adulthood. Lancet. 2008;371(9608):261-269.

6. Petrou $\mathrm{S}$. The economic consequences of preterm birth during the first 10 years of life. BJOG. 2005;112Suppl 1:10-15.

7. Tommiska V, Heinonen $\mathrm{K}$, Lehtonen L, et al. No improvement in outcome of nationwide extremely low birth weight infant populations between 1996-1997 and 1999-2000. Pediatrics. 2007;119(1):29-36.

8. Davidoff MJ, Dias T, Damus K, et al. Changes in the gestational age distribution among U.S. singleton births: impact on rates of late preterm birth, 1992 to 2002. Semin Perinatol. 2006;30(1):8-15.

9. Jasovic-Siveska E. Prevention and Prediction of Preterm Birth-Status Quo in the Last 50 Years. Reprod Syst Sex Disord. 2014;3(2):e117.

10. Escobar GJ, McCormick MC, Zupancic JA, et al. Unstudied Infants: Outcomes of moderately premature infants in the NICU. Arch Dis Child Fetal Neonatal Ed. 2006;91(4):F238-F244.

11. Hodek JM, von der Schulenburg JM, Mittendorf T. Measuring economic consequences of preterm birth-Methodological recommendations for the evaluation of personal burden on children and their caregivers. Health Econ Rev. 2011;1(1):6.

12. Meis PJ, Klebanoff M, Thom E, et al. Prevention of recurrent preterm delivery by 17 alpha-hydroxyprogesterone caproate. $N$ Engl J Med. 2003;348(24):2379-2385.

13. Lekovich J, Stewart J, Anderson S, et al. Placental malperfusion as a possible mechanism of preterm birth in patients with Mullerian anomalies. J Perinat Med. 2017;45(1):45-49.

14. Karahanoglu E, Altinboga O, Akpinar F, et al. Nifedipine increased fetoplacental perfusion. J Perinat Med. 2017;45(1):51-55.

15. Goldenberg RL, Culhane JF, Iams JD, et al. Epidemiology and causes of preterm birth. Lancet. 2008;371(9606):75-84.

16. Romero R, Espinoza J, Kusanovic JP, et al. The preterm parturition syndrome. BJOG. 2006;113Suppl3:17-42.

17. Baer RJ, Yang J, Chambers CD, et al. Risk of preterm birth among women according to change in partner. J Perinat Med. 2017;45(1):6370

18. McCormack RA, Doherty DA, Magann EF, et al. Antepartum bleeding of unknown origin in the second half of pregnancy and pregnancy outcomes. BJOG. 2008;115(11):1451-1457.
19. Chang HH, Larson J, Blencowe H, et al. Preventing preterm births: analysis of trends and potential reductions with interventions in 39 countries with very high human development index. Lancet. 2013;381(9862):223-234.

20. Romero R, Conde-Agudelo A, El-Refaie W, et al. Vaginal progesterone decreases preterm birth and neonatal morbidity and mortality in women with a twin gestation and a short cervix: an updated meta-analysis of individual patient data. Ultrasound Obstet Gynecol. 2017;49(3):303314.

21. Lamont RF. Infection in the prediction and antibiotics in the prevention of spontaneous preterm labour and preterm birth. BJOG. 2003;110(Suppl 20):71-75.

22. Koga K, Cardenas I, Aldo P, et al. Activation of TLR3 in the trophoblast is associated with preterm delivery. Am J Reprod Immunol. 2009;61(3):196-212.

23. Yoon BH, Kim YA, Romero R, et al. Association of oligohydramnios in women with preterm premature rupture of membranes with an inflammatory response in fetal, amniotic, and maternal compartments. Am J Obstet Gynecol. 1999;181(4):784-788.

24. Kemp MW. Preterm Birth, Intrauterine Infection, and Fetal Inflammation. Front Immunol. 2014;5:574.

25. Thorp JM. Predicting and preventing preterm birth. OBG Management. 2005;p.49-53.

26. Greco E, Lange A, Ushakov F, et al. Prediction of spontaneous preterm delivery from endocervical length at 11 to 13 weeks. Prenat Diagn. 2011;31(1):84-89.

27. Di Renzo Gc, Roura Lc, Facchinetti F, et al. Guidelines for the management of spontaneous preterm labor: identification of spontaneous preterm labor, diagnosis of preterm premature rupture of membranes, and preventive tools for preterm birth. J Matern Fetal Neonatal Med. 2011;24(5):659-667.

28. Tsoi E, Akmal S, Rane S, et al. Ultrasound assessment of cervical length in threatened preterm labor. Ultrasound Obstet Gynecol. 2003;21(6):552-555.

29. Polettini J, Cobo T, Kacerovsky M, et al. Biomarkers of spontaneous preterm birth: a systematic review of studies using multiplex analysis. $J$ Perinat Med. 2017;45(1):71-84.s

30. Berghella V, Ciardulli A, Rust OA, et al. Cerclage for sonographic short cervix in singleton gestations without prior spontaneous preterm birth: systematic review and meta-analysis of randomized controlled trials using individual patient-level data. Ultrasound Obstet Gynecol. 2017;50(5):569-577.

31. Society for Maternal Fetal Medicine Publications Committee. ACOG Committee Opinion number 419 October 2008 (replaces no. 291, November 2003). Use of progesterone to reduce preterm birth. Obstet Gynecol. 2008;112(4):963-965.

32. Likis FE, Edwards DR, Andrews JC, et al. Progestogens for preterm birth prevention: a systematic review and meta-analysis. Obstet Gynecol. 2012;120(4):897-907.

33. Ahn KH, Bae NY, Hong SC, et al. The safety of progesterone in the prevention of preterm birth: meta-analysis of neonatal mortality. $J$ Perinat Med. 2017;45(1):11-20.

34. Blencowe H, Cousens S, Oestergaard MZ, et al. National, regional, and worldwide estimates of preterm birth rates in the year 2010 with time trends since 1990 for selected countries: a systematic analysis and implications. Lancet. 2012;379:2162-2172. 